\title{
Anti-CD48/MMAE Antibody-drug Conjugate SGN-CD48A
}

National Cancer Institute

\section{Source}

National Cancer Institute. Anti-CD48/MMAE Antibody-drug Conjugate SGN-CD48A. NCI

Thesaurus. Code C148103.

An antibody-drug conjug ate (ADC) composed of an antibody targeting the cell surface antigen CD48 that is conjug ated to the microtubule-disrupting cytotoxic agent monomethyl auristatin E (MMAE), via a proprietary next-generation PEGylated glucuronide linker, with potential antineoplastic activity. Following intravenous administration, the antibody moiety of anti-CD48 ADC SGN-CD48A binds to CD48 on the surface of tumor cells. Following internalization of the ADC, the MMAE binds to tubulin and inhibits microtubule polymerization, which may result in G2/M phase cell cycle arrest and apoptosis in CD48-expressing tumor cells. CD48, a member of the signaling lymphocyte activation molecule (SLAM) family of immune cell receptors, is involved in Tcell activation and leukocyte trafficking. Additionally, CD48 is expressed on the surface of multiple myeloma cells at significantly higher levels than it is expressed on normal lymphocytes and monocytes. The linkage system in SGN-CD48A improves stability, reduces off-targ et uptake, and enables conjug ation of larger numbers of MMAE/antibody than other systems, resulting in increased specificity against CD48positive cells. 